\title{
FACULTY RESEARCH PRODUCTIVITY: Some Moderators of Associated Stressors
}

\author{
Robert T. Blackburn and Richard J. Bentley
}

Using person environment-fit theory, stress measures were developed on 894 faculty, a subgroup of the 3,972 faculty in the 1988 NCRIPTAL national survey. Within three institutional groups, three fields of study (humanities, natural sciences, and social sciences), three research output variables, and eight moderating variables (singly and collectively) were correlated (direct and partials) with two measures of stress. The findings lend support to the theoretical model. The outcomes also show that moderate levels of stress can be appreciably and significantly mitigated by some selected personal variables. The environmental variables were generally ineffective in moderating the correlations between stresses and productivity. Sex differences exist. Research self-competence is a strong modifier for both sexes and is exceptionally potent for women.

The past two decades have witnessed an appreciable growth in every college and university type in the number of faculty showing an increased interest in conducting research. Simultaneously, faculty report intensified institutional pressure to publish and secure external grants. The combined interests and pressures almost inevitably produce stress when faculty experience discrepancies between the amount of time they want to give to research differs from (1) what they actually are giving and/or (2) the fraction of time they believe their institution wants them to spend in research.'

While some amount of stress positively associates with scholarly performance, excessive stress becomes dysfunctional and needs mitigating (Pelz and Andrews, 1976). Consequently, it is important to know what variables moderate faculty stress. This study aims to provide knowledge on the relationship between stress, moderators, and research productivity. More specifically, this investigation;

1. Ascertains the direct effects of stressors on different kinds of research activity.

Address correspondence to: Robert T. Blackburn, 2017 School of Education, University of Michigan, Ann Arbor, 48109-1259. 
2. Tests a variety of psychological and environmental (organizational) variables in which theory predicts reduced stress and compares their partial correlations with direct effects of stress.

3. Explores stressors and moderator effects for gender, institutional type, and disciplinary differences

\section{THEORETICAL FRAMEWORK/RESEARCH LITERATURE}

The stress measures come from person-environment fit theory as developed by Argyris (1964), Locke (1969), Pelz and Andrews (1976), Lawler (1973), and others. Lewin (1951) provided the basis for the models in his work on motivation that took into account the interaction of the individual with the environment. Among others, Boberg (1982), Campbell (1973), French, Caplan, and Harrison (1982), Harrison (1976), and House (1972) have successfully tested the model. ${ }^{2}$ The key assumptions of the model are that stress results from a discrepancy between a person's motivations, abilities, or values and the corresponding opportunities, demands, or constraints of the workplace. The discrepancy-poor fit, lack of congruence-causes strain. However, both personal and contextual factors can moderate the effects of the stresses on the straings. Figure 1 from Harrison depicts the P-E fit model.

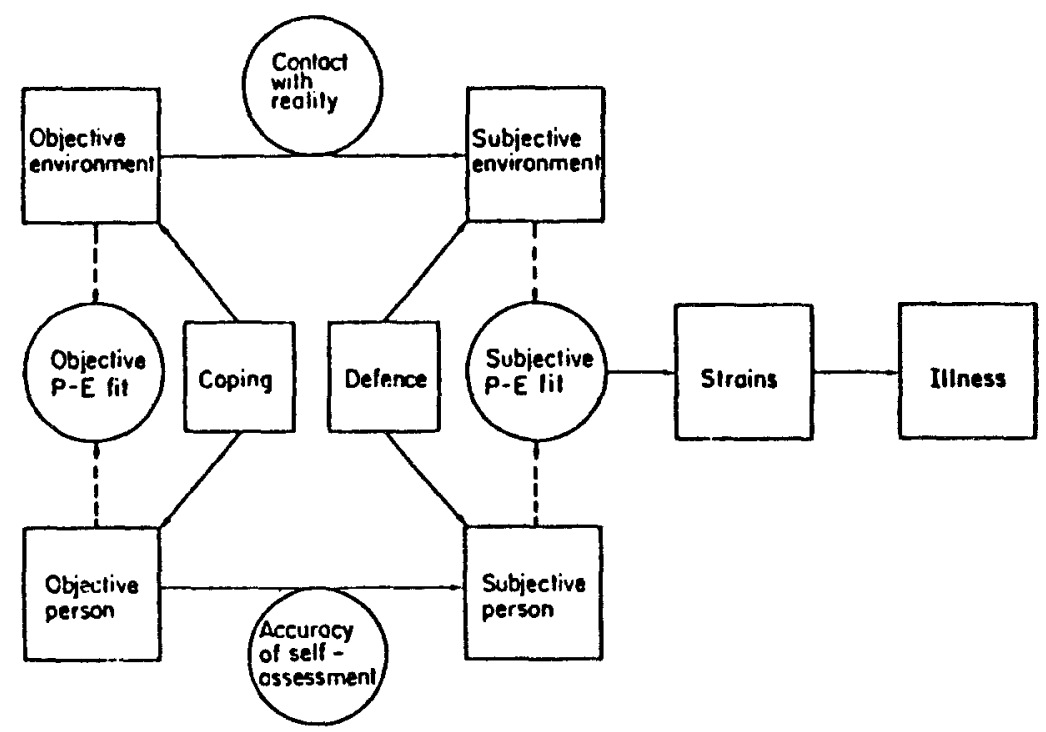

FIG. 1. A model describing the effects of psychosocial stress in terms of fit between the person and the environment. Concepts within circles are discrepancies between the two adjoining concepts. Solid lines indicate causal effects. Broken lines indicate contributions to interaction effects Source: Harrison, 1980, p. 176. 
Our study is completely in the subjective domain. We ascertained no objective measures of institutional expected work effort or actual faculty research effort. The larger theoretical model for predicting faculty productivity comes from Lawrence and colleagues (1989). The specific model employed for the analyses conducted here follows Blackburn, Horowitz, Edington, and Kloss (1987, p. 32). See Figure 2.

Simply put, job-related stresses (differences between motivated preferred effort to give to research activities and perceived institutional expectations) produce strains (unsatisfactory research output). The discrepancy can be in either direction. For example, I can want more time for research than I see my institution rewards. In fact, I can suspect that my institution may penalize me when it sees I am spending long hours in research but less time with stuents.

On the other side, I might work in an environment that wants more research of me than I know I can produce. I perceive excessive demands. From either perspective, these stress producers predict reduced research output. Result: I will fail my tenure review. At the same time, some personal and environmental attributes and conditions are hypothesized as being capable of modifying the deleterious effects of stress.

The stress indicators (see below) are modifications of those used by Clark and Blackburn (1973) and Boberg (1982). We also selected variables on the basis of other faculty stress studies (e.g., Gmelch, Lovrich, and Wilke, 1984; Gmelch, Wilke, and Lovrich, 1986; Keinan and Perlberg, 1987; and Rutheram and Weiner, 1983).

We know women publish less than men (Zuckerman, 1991). Numerous hypotheses have been advanced to explain the differences-biological, cultural, structural, and psychological. (See, e.g., the chapters in Zuckerman, Cole, and Bruer, 1991.) None, however, has included possible differential effects of stress. Moreover, neither Boberg nor Gmelch et al. explored differences in stress by sex. Blackburn et al.'s subjects were all men. Dey (1990), however, found several differences between men and women on some factor items, with women showing stronger loadings. He also found higher percentages of women rating different stress areas as extensive sources of stress. However, since there is no linking the stress indicators to research output, it is important to see if stress differences and their possible modifications differ between the sexes. We therefore conducted separate analyses for female and male faculty.

We also introduced controls for institutional type and academic field since both have been demonstrated to be related to faculty publications (Bentley and Blackburn, 1991). On average, faculty in research universities publish more than do faculty in other institutional types. While faculty in all fields write both articles and books, the articles in scholarly journals characterize the sciences and University Press books represent the principal scholarship of humanists (Bieber and Blackburn, 1989). That is, there are differences both in kind and in amount. One might expect stress to publish to be higher in the research univer- 
बิ

$\dot{0}$
0
0
0
0
0
$\dot{b}$

ָָ

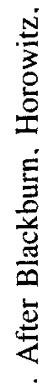

$\frac{\overline{8}}{\frac{8}{8}}$

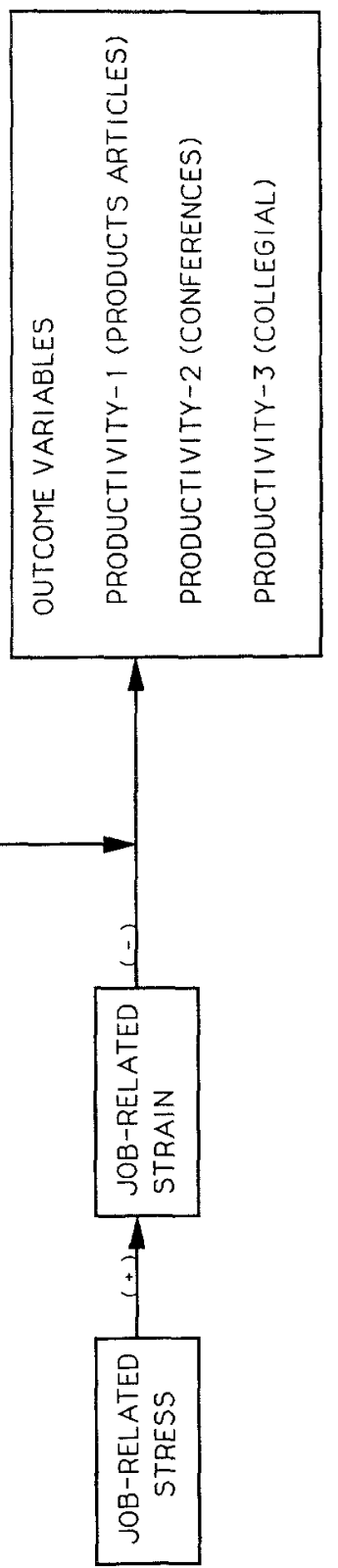


sities for rewards there are closely tied to publications. On the other hand, the research emphasis has been spreading to the comprehensive and liberal arts institutions and one can hypothesize stress on faculty in these settings as well. Furthermore, stress can affect all regardless of location or discipline. By controlling for these two aspects, our analysis is not jeopardized by specific institutional stress differences.

\section{DATA SOURCES}

Data from Faculty at Work, a national survey conducted by the National Center for Research to Improve Postsecondary Teaching and Learning, constitute the data source. The stratified, random sample was drawn in proportion to the distribution of professors across nine Carnegie (1987) classification categories. It corresponds to the national distribution of faculty members across institutional types and is the most representative of the 15 national surveys conducted thus far (Bentley, Blackburn, and Bieber, 1991). The survey took place between November 1987 and January 1988 and involved full-time faculty with regular appointments in eight disciplines: English and history (humanities); biology, chemistry, and mathematics (natural sciences); and political science, psychology, and sociology (social sciences). These eight largest disciplines constitute a cross section of the liberal arts and sciences found at nearly every college and university. The survey was distributed to 8,130 faculty members in 236 institutions and was completed by 3,972 respondents (49.7 percent response rate).

\section{METHODOLOGY}

We controlled for the larger work environment by analyzing data within aggregated institutional (Carnegie, 1987) types, ones created in order to maintain adequate $N$ 's for analyses. We grouped institutional types on the basis of their overall emphasis and support for research. (See Results section below.)

We derived two stress measures (independent variables) from taking the differences between (1) faculty reported percentage of effort they give to research and the percentage of effort they wish to give to that role and (2) the percentage of effort they believe their institution wants them to give to research and the percentage of effort they prefer to give. These stress indicators assume that strains occur when one (a) is not doing what one prefers and/or (b) is allocating effort differently from what one believes the rewarding organization wants.

The study's approach follows the successful studies of Clark and Blackburn (1973) and Boberg (1982) and differs from the methods used by Gmelch et al. (1984, 1986) and Dey (1990). Both Gmelch and Dey directly ask faculty to tell 
how stressful a variety of situations are (e.g., teaching load, committee work, marital friction), tally and/or factor the responses, and report their findings. ${ }^{3}$

As we designed the Faculty at Work survey instrument from the conceptual framework of Lawrence et al., we introduced both self- and social-knowledge variables. Preferred effort for research is a self knowledge variable; perceived expectation of the institution is a social knowledge variable. By including the two items in this way we were able to introduce an additional personal variable, an indicator of stress. With the addition of percent of actual effort we had a second way of estimating the stress facuty were undergoing. The differences between actual and preferred and between actual and perceived institutional preference provide two stress measures.

The direct method has high face validity, but low reliability. Item responses can fluctuate from day to day, even during a day, and hence are less stable indicators of stress. For example, hours in committee meetings can contribute to a high level of stress during one week but drain few precious hours at another time. The direct questions are also susceptible to a "transparency" effect. Faculty ritualistically must complain about their teaching load and can feel obliged to identify it as a source of stress when in fact it may not be one of the principal causes. The consequences can be an inaccurate interpretation of teaching load as a stressor.

The method used in this study has a demonstrated high retest reliability (Blackburn and Mackie, 1992) but a more debatable face validity. Low reliability, however, destroys the use of an indicator-no matter how valid. The time allocation discrepancy measure is also a continuous rather than a categorical variable and has this virtue as well when it comes to statistical analyses

Four personal moderating variables were scales (or an item in one instance): the self and social knowledge variables were taken from Blackburn et al. (1991). They were the ones that were the strongest predictors of research productivity and therefore most likely to mitigate stress:

1. Interest in reserach (Int-Res): one item-respondent is more interested in research than in teaching.

2. Self-competence in research (Self-Comp): two items-how characteristic of the respondent is (1) publishing and (2) obtaining grants.

3. Self-efficacy (Self-Eff): two items-how much influence a respondent has in (1) getting something published and (2) securing extra funding for travel to conferences.

4. Personal characteristics (Prsnlty): four items-how characteristic of the respondent is (1) ambitious, (2) persevering, (3) competitive, and (4) highly committed to research.

Four environmental moderating variables were scales or items: 
1. Environmental support (Support): four items-faculty at their institution are (1) primarily oriented to professional accomplishment, (2) more committed to teaching than to doing disciplinary research (reverse scored), (3) have colleagues who know my specialty well enough to assist and critically review my scholarly work, and (4) say support services are available for conducting my scholarship.

2. Assistance (Assist): two-item, continuous scale: (1) number of hours per week of student assistance and (2) number of hours per week of clerical assistance.

3. Institutional grants (Ingrant): Faculty member has had an institutional grant in the last 12 months.

4. External grants (Exgrant): Faculty member had at least one external (federal, governmental, foundation, industrial, or other) grant in the last 12 months.

The three dependent/productivity variables are:

1. Productivity-1 (Prodl): While identifying a variety of things faculty produce, each of these seven intercorrelated items is a written scholarly product. The scale is taken directly from Blackburn et al. (1991). The items have an average retest reliability of .81 , the highest of any item set in the NCRIPTAL study (Blackburn and Mackie, 1992). As a seven-item scale, it has high internal reliability as well. ${ }^{4}$ The seven-item scale records how often during the prior two years the subject had:

(a) submitted an article for publication in an academic or professional journal;

(b) published chapters in a book;

(c) submitted a research proposal to a governmental or private agency;

(d) written a research report for an agency, institutions, or other group;

(e) published scholarly articles;

(f) submitted external grant proposals;

(g) had professional writings published or had writings accepted for publication.

2. Productivity-2 $(\operatorname{Prod} 2)$ : These are presentations. The scale is composed of two items:

(a) how often the subject had presented her/his ongoing work on campus during the past year;

(b) how often during the past two years the faculty member had made a presentation at a professional conference.

3. Productivity-3 (Prod3): These are conversations regarding research. The collegial scale is composed of two items:

(a) how many times in the past year at professional meetings the subject 
had informal conversations with colleagues about research;

(b) how many times in the past year the subject had telephone conversations with colleagues to discuss her/his scholarly work.

\section{RESULTS}

Table 1 displays selected characteristics of the population by institutional type and for the three institutional groups used in the statistical analyses. Only minor differences exist by sex, career age (difference between year of highest degree and date of data collection), sex, and rank (five of the first six entries). Appreciably more faculty in the first category received their highest degree from a Research-I university, the places that most thoroughly socialize their graduate students into the research role.

The last three entries-interest in research (the percentage of the faculty who responded "primarily interested in research" plus those who responded "interested both in research and teaching, but more so in research" versus the other two categories that favor teaching), the percentage of their weekly work effort they wish to give to research, and the percentage of the work week they believe their administration wants them to give to research-are essentially identical for categories II and III institutions. However, both differ appreciably from faculty in category I. Faculty at Research-I and -II universities express almost twice as high an interest in research; their preferred percentage effort and amount they perceived their institution desires are about half again as much as faculty in the four other Carnegie types. The differences between preferred and believed percentages are small for all three categories, on average. Appreciable individual variation exists so that discrepancies cover a wide span and allow for testing stress effects.

Tables 2-6 display the two stress measure data: (a) Stress-1, the difference between a faculty member's preferred and actual effort given to research and (b) Stress-2, the difference between a faculty member's perceived institutional preference and her or his personal preference for percentage of effort given to research. Data are shown for personal moderators by institutional categories (Research-I and -II universities in Tables 2 and 5, Doc-I and LA-I in Table 3, and Doc-II and Comp-I in Table 4) and by the three disciplinary areas-natural sciences (Nat Sci), humanities (Human), and social sciences (Soc Sci). Table 6 contains the comparable data for environmental variables for research universities.

Within each of these categories, each productivity measure first reports the direct effect of both stresses; then with each moderator independently; and last with all moderators entered. Results for men, women, and the total sample are shown separately.

To begin, Table 2 shows that for the total group (males, females, and com- 


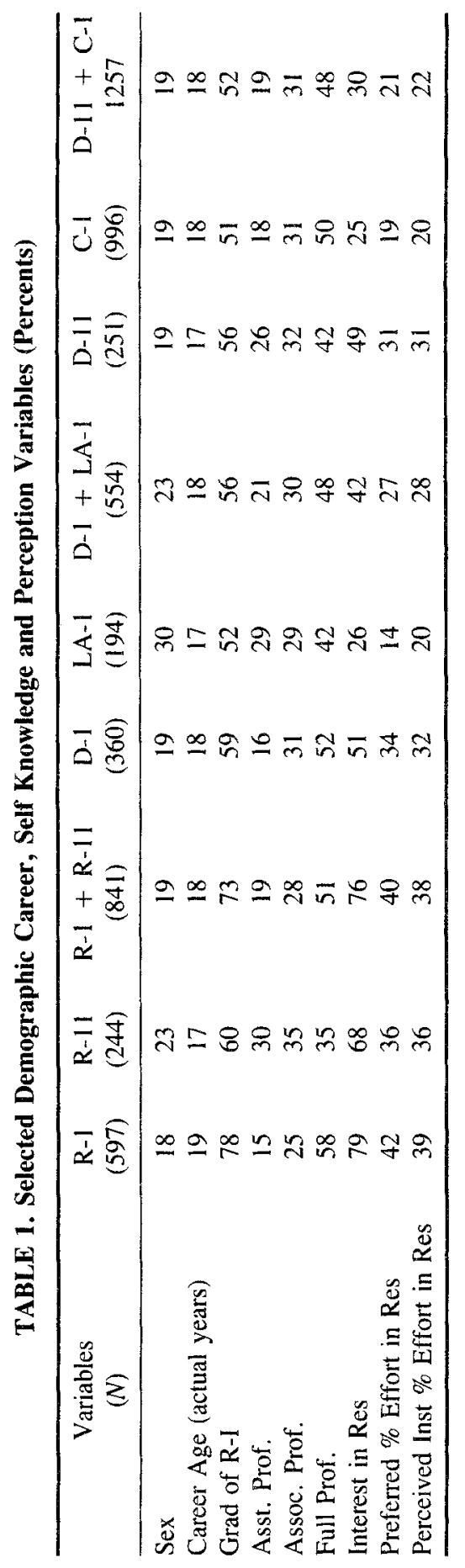


TABLE 2. Direct Effects and Partial Correlations (Research I \& II)

\begin{tabular}{|c|c|c|c|c|c|c|c|c|}
\hline \multirow{5}{*}{$\begin{array}{l}\text { Nat } \\
\text { Sci } \\
\text { Prod l }\end{array}$} & \multirow{5}{*}{$\begin{array}{l}\text { Nat } \\
\text { Sci } \\
\text { Prod2 }\end{array}$} & & \multicolumn{3}{|c|}{ Stress 1} & \multicolumn{3}{|c|}{ Stress 2} \\
\hline & & \multirow[b]{2}{*}{$\operatorname{Max} N^{\prime} \mathrm{s}$} & \multirow{2}{*}{$\frac{\text { Male }}{(266)}$} & \multirow{2}{*}{$\frac{\text { Female }}{(37)}$} & \multirow{2}{*}{$\frac{\text { All }}{(303)}$} & \multirow{2}{*}{$\frac{\text { Male }}{(266)}$} & \multirow{2}{*}{$\frac{\text { Female }}{(37)}$} & \multirow{2}{*}{$\frac{\text { All }}{(303)}$} \\
\hline & & & & & & & & \\
\hline & & Direct & $-0.48 * *$ & $-0.51 * *$ & $-0.48 * *$ & $0.34 * *$ & $0.41 *$ & 0.34 \\
\hline & & Int-Res & $-0.37 * *$ & -0.18 & $-0.35^{* *}$ & $0.19 * *$ & 0.02 & 0.17 \\
\hline & & Self-Comp & $-0.23 * *$ & -0.07 & $-0.21 * *$ & 0.09 & 0.31 & 0.11 \\
\hline & & Self-Eff & $-0.46^{* *}$ & $-0.44^{*}$ & $-0.44 * *$ & $0.31 * *$ & $0.34^{*}$ & 0.31 \\
\hline & & Prsnlty & $-0.37 * *$ & $-0.36 *$ & $-0.36 * *$ & $0.22^{* *}$ & 0.24 & 0.21 \\
\hline & & All & $-0.21 * *$ & 0.13 & $-0.19^{* *}$ & 0.05 & 0.19 & 0.06 \\
\hline \multirow[t]{6}{*}{ Prod2 } & Prod2 & Direct & $-0.33 * *$ & -0.26 & $-0.33 * *$ & $0.34^{* *}$ & 0.06 & 0.31 \\
\hline & & Int-Res & $-0.20 * *$ & -0.07 & $-0.19 * *$ & $0.16^{* *}$ & -0.24 & 0.12 \\
\hline & & Self-Comp & -0.11 & 0.14 & -0.09 & $0.13^{*}$ & -0.17 & 0.10 \\
\hline & & Self-Eff & $-0.30 * *$ & -0.20 & $-0.29 * *$ & $0.28 * *$ & -0.03 & 0.25 \\
\hline & & Prsnlty & $-0.22 * *$ & -0.15 & $-0.21 * *$ & $0.21 * *$ & -0.11 & 0.18 \\
\hline & & All & -0.05 & 0.17 & -0.04 & 0.07 & -0.17 & 0.04 \\
\hline \multirow[t]{6}{*}{ Prod3 } & Prod3 & Direct & $-0.43^{* *}$ & $-0.38^{*}$ & $-0.42 * *$ & $0.42^{* *}$ & 0.22 & 0.40 \\
\hline & & Int-Res & $-0.30^{* *}$ & -0.21 & $-0.29 * *$ & $0.27^{* *}$ & 0.01 & 0.25 \\
\hline & & Self-Comp & $-0.12^{*}$ & -0.01 & $-0.12^{*}$ & $0.19 * *$ & 0.09 & 0.19 \\
\hline & & Self-Eff & $-0.39 * *$ & -0.31 & $-0.38 * *$ & $0.39 * *$ & 0.18 & 0.37 \\
\hline & & Prsnlty & $-0.30 * *$ & -0.19 & $-0.29 * *$ & $0.31 * *$ & 0.03 & 0.28 \\
\hline & & All & -0.10 & -0.07 & -0.10 & $0.17^{* *}$ & 0.15 & 0.17 \\
\hline Human & Human & $\operatorname{Max} N$ 's & (171) & (61) & $(233)$ & $(171)$ & $(61)$ & (233) \\
\hline \multirow[t]{6}{*}{ Prodl } & Prodl & Direct & $-0.25^{* *}$ & $-0.31^{*}$ & $0.25^{* *}$ & $0.32 * *$ & $0.32 * *$ & 0.29 \\
\hline & & Int-Res & -0.12 & -0.23 & -0.13 & 0.12 & 0.09 & 0.10 \\
\hline & & Self-Comp & $-0.19^{*}$ & -0.23 & $-0.18^{*}$ & $0.17 *$ & 0.10 & 0.13 \\
\hline & & Self-Eff & $-0.23 * *$ & $-0.34 *$ & $-0.26^{* *}$ & $0.29 * *$ & $0.30^{*}$ & 0.28 \\
\hline & & Prsnlty & $-0.16^{*}$ & $-0.32 *$ & $-0.19 * *$ & $0.18^{*}$ & 0.23 & 0.18 \\
\hline & & All & -0.13 & -0.15 & -0.13 & 0.12 & 0.00 & 0.07 \\
\hline \multirow[t]{6}{*}{ Prod2 } & Prod2 & Direct & $-0.29 * *$ & 0.00 & $-0.18^{* *}$ & $0.26^{* *}$ & 0.02 & 0.18 \\
\hline & & Int-Res & $-0.19^{*}$ & 0.03 & -0.10 & 0.12 & -0.06 & 0.04 \\
\hline & & Self-Comp & $-0.25^{* *}$ & 0.10 & -0.12 & $0.19^{*}$ & -0.16 & 0.06 \\
\hline & & Self-Eff & $-0.27 * *$ & 0.03 & $-0.17^{*}$ & $0.27^{* *}$ & -0.04 & 0.15 \\
\hline & & Prsnlty & $-0.24 * *$ & 0.03 & -0.13 & $0.20^{*}$ & -0.06 & 0.08 \\
\hline & & All & $-0.20^{*}$ & 0.12 & -0.11 & $0.16^{*}$ & -0.14 & 0.04 \\
\hline \multirow[t]{6}{*}{ Prod3 } & Prod3 & Direct & $-0.25^{* *}$ & $-0.40^{* *}$ & $-0.22^{* *}$ & $0.32 * *$ & $0.33 * *$ & 0.28 \\
\hline & & Int-Res & $-0.16^{*}$ & $-0.33^{*}$ & -0.12 & 0.11 & 0.23 & 0.09 \\
\hline & & Self-Comp & $-0.18^{*}$ & $-0.28^{*}$ & -0.12 & 0.11 & 0.11 & 0.06 \\
\hline & & Self-Eff & $-0.20^{*}$ & $-0.47^{* *}$ & $-0.20^{* *}$ & $0.21 *$ & $0.38 * *$ & 0.20 \\
\hline & & Prsnlty & -0.10 & $-0.37 * *$ & -0.10 & 0.02 & $0.27^{*}$ & 0.03 \\
\hline & Soc & All & -0.12 & $-0.33^{*}$ & -0.12 & 0.07 & 0.15 & 0.05 \\
\hline Sci & Sci & $\operatorname{Max} N^{\prime} \mathrm{s}$ & (239) & (65) & $(308)$ & $(239)$ & $(65)$ & (308) \\
\hline Prod 1 & Prod 1 & Direct & $-0.32 * *$ & $-0.53^{* *}$ & $-0.35 * *$ & $0.30 * *$ & $0.52^{* * *}$ & 0.32 \\
\hline
\end{tabular}




\begin{tabular}{|c|c|c|c|c|c|c|c|c|}
\hline & & Int-Res & $-0.13^{*}$ & $-0.41 * *$ & $-0.18 * *$ & $0.13^{*}$ & $0.40^{* *}$ & 0.17 \\
\hline & & Self-Comp & -0.12 & $-0.25^{*}$ & $-0.13^{*}$ & $0.14^{*}$ & $0.30 *$ & 0.15 \\
\hline & & Self-Eff & $-0.27 * *$ & $-0.41^{*}$ & $-0.29 * *$ & $0.28^{* *}$ & $0.49^{* *}$ & 0.30 \\
\hline & & Prsnlty & $-0.20 * *$ & $-0.44 * *$ & $-0.23 * *$ & $0.19 * *$ & $0.46 * *$ & 0.22 \\
\hline & & All & -0.09 & -0.09 & -0.09 & 0.10 & 0.19 & 0.11 \\
\hline Prod2 & Prod2 & Direct & $-0.21 * *$ & $-0.28 *$ & $-0.22 * *$ & $0.14 *$ & 0.16 & 0.16 \\
\hline & & Int-Res & -0.09 & $-0.30^{*}$ & $-0.13^{*}$ & 0.04 & 0.17 & 0.06 \\
\hline & & Self-Comp & -0.04 & -0.07 & -0.04 & 0.00 & -0.09 & -0.01 \\
\hline & & Self-Eff & $-0.16^{*}$ & -0.08 & $-0.16 * *$ & $0.13 *$ & 0.05 & 0.11 \\
\hline & & Prsnlty & -0.06 & -0.18 & -0.08 & 0.00 & 0.03 & 0.00 \\
\hline & & All & 0.04 & 0.04 & -0.04 & -0.02 & -0.04 & -0.03 \\
\hline Prod3 & Prod3 & Direct & $-0.23^{* *}$ & $-0.34 * *$ & $-0.24 *$ & $0.22 * *$ & -0.02 & 0.21 \\
\hline & & Int-Res & -0.06 & $-0.37 * *$ & $-0.12^{*}$ & 0.11 & $-0.29 *$ & 0.11 \\
\hline & & Self-Comp & -0.03 & -0.14 & -0.05 & 0.08 & -0.13 & 0.05 \\
\hline & & Self-Eff & $-0.17^{*}$ & -0.13 & $-0.17 * *$ & $0.22 * *$ & -0.03 & 0.18 \\
\hline & & Prsnlty & -0.06 & -0.14 & -0.07 & 0.08 & -0.12 & 0.05 \\
\hline & & All & 0.00 & -0.12 & -0.01 & 0.04 & -0.04 & 0.01 \\
\hline
\end{tabular}

${ }^{* *} p<.01 ;{ }^{*} p<.05$

bined sexes) the signs for all of the direct effects are as expected-negative correlations for Stress-1 since preferred time is less than actual time and positive correlations for Stress-2 when the perceived institutional desired effort is greater than faculty want to give. By way of illustration, in the top row for the combined group of natural science faculty, the direct correlations for the product (Prodl) productivity outcome measure are -0.48 and 0.34 , respectively, both at $p<.01$. The values have small sex differences (e.g., -.48 for men and -.51 for women for Stress-1).

The coefficients generally are statistically significant and moderately high. All 18 (two stresses by three outcomes by three disciplines) of the direct correlations for the entire population are at $p<.01$ for research universities. The numbers of significant $r$ 's are somewhat less for the other two institutional categories (Tables 3 and 4 ) where faculty experience a less intense research climate.

Overall, there are not great differences between disciplinary fields, the three productivity measures, and the type of stress. The direct effects are stronger in the natural sciences and about the same in the humanities and the social sciences. The correlations tend to be slightly higher for the product output measure (Prod1) than they are for the conference (Prod2) and conversational/collegial (Prod3) indicators of research behavior as might be expected. ${ }^{5}$ Institutions reward publications, not talk about them. Since both stress measures involve a gap between what the faculty member wants to do and other constraints, they correlate. Hence, it is not surprising that similar patterns appear in many instances. 
TABLE 3. Direct Effects and Partial Correlations (Doc I \& Liberal Arts I)

\begin{tabular}{|c|c|c|c|c|c|c|c|}
\hline \multirow{2}{*}{ Nat } & & \multicolumn{3}{|c|}{ Stress 1} & \multicolumn{3}{|c|}{ Stress 2} \\
\hline & & Male & Female & All & Male & Female & All \\
\hline Sci & $\operatorname{Max} N^{\prime} \mathrm{s}$ & $(127)$ & (29) & $(157)$ & $(130)$ & (29) & $(161)$ \\
\hline \multirow[t]{6}{*}{ Prodl } & Direct & $-0.20 * *$ & 0.03 & -0.12 & 0.12 & 0.12 & 0.12 \\
\hline & Int-Res & -0.08 & 0.06 & -0.03 & $-0.17 *$ & -0.26 & $-0.17 *$ \\
\hline & Self-Comp & -0.02 & 0.10 & 0.02 & -0.08 & 0.03 & -0.05 \\
\hline & Self-Eff & -0.14 & 0.10 & -0.08 & 0.06 & 0.08 & 0.07 \\
\hline & Prsnlty & -0.05 & 0.01 & 0.00 & -0.04 & 0.07 & -0.04 \\
\hline & All & 0.01 & 0.08 & 0.08 & $-0.20^{*}$ & -0.04 & -0.02 \\
\hline \multirow[t]{6}{*}{ Prod2 } & Direct & -0.10 & 0.05 & -0.07 & -0.05 & $0.08^{*}$ & -0.02 \\
\hline & Int-Res & 0.00 & 0.05 & 0.00 & $-0.27 * *$ & -0.08 & $-0.13 * *$ \\
\hline & Self-Comp & 0.05 & 0.05 & 0.04 & $-0.25^{* *}$ & 0.04 & $-0.19 * *$ \\
\hline & Self-Eff & -0.03 & 0.05 & -0.02 & $-0.15^{*}$ & 0.06 & -0.11 \\
\hline & Prsnlty & 0.02 & -0.01 & 0.03 & $-0.21 * *$ & 0.06 & $-0.17^{*}$ \\
\hline & All & 0.06 & 0.00 & -0.05 & $-0.29 * *$ & 0.06 & -0.06 \\
\hline \multirow[t]{6}{*}{ Prod3 } & Direct & $-0.25^{* *}$ & 0.02 & $-0.20^{* *}$ & 0.07 & 0.05 & 0.08 \\
\hline & Int-Res & $-0.16^{*}$ & 0.07 & -0.12 & -0.11 & -0.19 & -0.10 \\
\hline & Self-Comp & -0.08 & 0.10 & -0.06 & -0.12 & 0.04 & -0.07 \\
\hline & Self-Eff & $-0.18^{*}$ & 0.06 & $-0.14^{*}$ & 0.01 & 0.07 & 0.04 \\
\hline & Prsnlty & -0.12 & 0.01 & -0.08 & $-0.04 * *$ & 0.07 & -0.03 \\
\hline & All & -0.07 & 0.02 & -0.03 & -0.15 & 0.02 & -0.02 \\
\hline Human & $\operatorname{Max} N$ 's & (94) & (33) & (127) & $(98)$ & $(40)$ & $(138)$ \\
\hline \multirow[t]{6}{*}{ Prod 1} & Direct & $-0.33^{* *}$ & -0.26 & $-0.32 * *$ & $0.44^{* *}$ & -0.26 & $0.34 * *$ \\
\hline & Int-Res & $-0.26^{* *}$ & 0.00 & $-0.20^{*}$ & $0.24 * *$ & -0.17 & 0.14 \\
\hline & Self-Comp & -0.14 & 0.13 & -0.09 & $0.19^{*}$ & -0.08 & 0.12 \\
\hline & Self-Eff & $-0.31 * *$ & -0.25 & $-0.28^{* *}$ & $0.39 * *$ & 0.04 & $0.30^{* *}$ \\
\hline & Prsnlty & $-0.20^{*}$ & -0.15 & $-0.19^{*}$ & $0.24 * *$ & 0.05 & $0.20^{*}$ \\
\hline & All & -0.13 & 0.17 & -0.07 & 0.12 & -0.21 & 0.07 \\
\hline \multirow[t]{6}{*}{ Prod2 } & Direct & $-0.20^{*}$ & 0.07 & -0.14 & $0.22 * *$ & 0.02 & $0.16^{*}$ \\
\hline & Int-Res & -0.13 & 0.21 & -0.06 & 0.07 & -0.16 & 0.02 \\
\hline & Self-Comp & -0.04 & $0.36^{*}$ & 0.04 & 0.00 & -0.17 & -0.03 \\
\hline & Self-Eff & -0.14 & 0.12 & 0.04 & 0.14 & -0.11 & 0.09 \\
\hline & Prsnlty & -0.08 & 0.17 & -0.03 & 0.05 & -0.09 & 0.02 \\
\hline & All & 0.00 & $0.34 *$ & 0.06 & -0.05 & -0.15 & -0.05 \\
\hline \multirow[t]{6}{*}{ Prod3 } & Direct & $-0.17 * *$ & -0.23 & $-0.18^{*}$ & $0.25^{* *}$ & 0.19 & $0.23 * *$ \\
\hline & Int-Res & -0.11 & -0.02 & -0.09 & 0.10 & 0.08 & 0.10 \\
\hline & Self-Comp & -0.04 & -0.09 & -0.05 & 0.06 & 0.20 & 0.11 \\
\hline & Self-Eff & -0.14 & -0.22 & -0.15 & $0.19 *$ & 0.23 & $0.20 * *$ \\
\hline & Prsnlty & $-0.08 * *$ & -0.10 & -0.08 & 0.10 & 0.23 & 0.13 \\
\hline & All & -0.01 & 0.02 & -0.01 & 0.01 & 0.12 & 0.04 \\
\hline Sci & $\operatorname{Max} N$ 's & $(111)$ & (32) & $(144)$ & $(110)$ & (31) & $(142)$ \\
\hline
\end{tabular}




\begin{tabular}{llrlcccc} 
Prod1 & Direct & -0.10 & $-0.29^{*}$ & -0.13 & $0.22^{* *}$ & $0.32 *$ & $0.22^{* *}$ \\
& Int-Res & 0.08 & -0.13 & 0.05 & -0.08 & 0.23 & -0.05 \\
& Self-Comp & 0.07 & -0.10 & 0.04 & 0.00 & 0.17 & 0.01 \\
& Self-Eff & -0.13 & -0.18 & -0.13 & $0.23^{* *}$ & $0.34^{*}$ & $0.22^{* *}$ \\
& Prsnlty & -0.07 & -0.21 & $-0.17^{* *}$ & $0.16^{*}$ & $0.38^{*}$ & $0.18^{*}$ \\
& All & 0.12 & -0.04 & 0.10 & -0.09 & 0.04 & -0.10 \\
Prod2 & Direct & -0.10 & -0.22 & -0.12 & 0.13 & 0.26 & $0.17^{*}$ \\
& Int-Res & 0.07 & -0.06 & 0.03 & -0.11 & 0.15 & -0.03 \\
& Self-Comp & 0.05 & -0.01 & -0.03 & -0.04 & 0.04 & -0.01 \\
& Self-Eff & -0.07 & -0.05 & -0.07 & $0.10^{*}$ & 0.13 & 0.12 \\
& Prsnlty & -0.03 & -0.10 & -0.08 & 0.06 & 0.21 & 0.10 \\
& All & 0.09 & 0.02 & 0.06 & -0.12 & -0.01 & -0.06 \\
Prod3 & Direct & $-0.19^{*}$ & $-0.38^{*}$ & $-0.23^{* *}$ & $0.24^{* *}$ & $0.41^{* *}$ & $0.28^{* *}$ \\
& Int-Res & 0.00 & $-0.43^{*}$ & -0.11 & -0.03 & $0.46^{* *}$ & 0.12 \\
& Self-Comp & -0.06 & $-0.42^{*}$ & -0.14 & 0.10 & $0.38^{*}$ & $0.18^{*}$ \\
& Self-Eff & $-0.18^{*}$ & $-0.43^{*}$ & $-0.23^{* *}$ & $0.25^{* *}$ & $0.45^{* *}$ & $0.30^{* *}$ \\
& Prsnlty & -0.14 & $-0.47^{* *}$ & $-0.12^{*}$ & $0.20^{*}$ & $0.51^{* *}$ & $0.27^{* *}$ \\
& All & 0.01 & $-0.43^{*}$ & -0.09 & -0.03 & $0.40^{*}$ & 0.11 \\
\hline
\end{tabular}

$* * p<.01 ; * p<.05$

TABLE 4. Direct Effects and Partial Correlations (Doc II and Comp I)

\begin{tabular}{|c|c|c|c|c|c|c|c|}
\hline \multirow[b]{2}{*}{ Nat } & & \multicolumn{3}{|c|}{ Stress 1} & \multicolumn{3}{|c|}{ Stress 2} \\
\hline & & Male & Female & All & Male & Female & All \\
\hline $\mathrm{Sci}$ & $\operatorname{Max} N$ 's & $(334)$ & $(56)$ & $(402)$ & (334) & $(57)$ & $(425)$ \\
\hline \multirow[t]{6}{*}{ Prod 1} & Direct & $-0.33^{* *}$ & $-0.36 * *$ & $-0.33^{* *}$ & $0.26^{* *}$ & $0.29 *$ & $0.26^{* *}$ \\
\hline & Int-Res & -0.05 & -0.13 & -0.06 & -0.04 & 0.01 & -0.05 \\
\hline & Self-Comp & 0.00 & -0.04 & 0.00 & 0.00 & 0.09 & 0.01 \\
\hline & Self-Eff & $-0.28 * *$ & $-0.30 *$ & $-0.28 * *$ & $0.26^{* *}$ & $0.24^{*}$ & $0.25 * *$ \\
\hline & Prsnlty & $-0.15^{* *}$ & -0.20 & -0.16 & $0.14^{* *}$ & 0.16 & $0.13^{* *}$ \\
\hline & All & 0.08 & 0.04 & 0.08 & $-0.11^{*}$ & -0.02 & $-0.10^{*}$ \\
\hline \multirow[t]{6}{*}{ Prod2 } & Direct & $-0.27 * *$ & $-0.27 *$ & $-0.29 * *$ & $0.20 * *$ & $0.25^{*}$ & $0.21 * *$ \\
\hline & Int-Res & $-0.11^{*}$ & $-0.27 *$ & $-0.13 * *$ & -0.03 & 0.07 & -0.01 \\
\hline & Self-Comp & -0.07 & -0.22 & -0.09 & -0.01 & 0.13 & 0.01 \\
\hline & Self-Eff & $-0.24 * *$ & $-0.38^{* * *}$ & $-0.26^{* *}$ & $0.15^{* *}$ & $0.25^{*}$ & $0.17 * *$ \\
\hline & Prsnlty & $-0.15^{* * *}$ & $-0.24^{*}$ & $-0.16^{* *}$ & 0.06 & 0.11 & 0.07 \\
\hline & All & -0.03 & -0.20 & -0.05 & -0.08 & 0.11 & -0.06 \\
\hline \multirow[t]{6}{*}{ Prod3 } & Direct & $-0.34^{* *}$ & 0.12 & $-0.30 * *$ & $0.29 * *$ & 0.02 & $0.25 * *$ \\
\hline & Int-Res & $-0.16^{* *}$ & 0.12 & $-0.12 * *$ & 0.06 & -0.13 & 0.03 \\
\hline & Self-Comp & $-0.13^{* *}$ & 0.15 & $-0.09^{*}$ & $0.10^{*}$ & -0.07 & 0.07 \\
\hline & Self-Eff & $-0.29 * *$ & 0.00 & $-0.26 * *$ & $0.25 * *$ & 0.02 & $0.21 * *$ \\
\hline & Prsnlty & $-0.19^{* *}$ & 0.08 & $-0.15 * *$ & $0.14^{* *}$ & -0.04 & $0.11 *$ \\
\hline & All & -0.06 & 0.20 & -0.03 & 0.00 & -0.14 & -0.02 \\
\hline
\end{tabular}


TABLE 4. Direct Effects and Partial Correlations (Doc II and Comp I) (Contd.)

\begin{tabular}{|c|c|c|c|c|c|c|c|}
\hline & & \multicolumn{3}{|c|}{ Stress 1} & \multicolumn{3}{|c|}{ Stress 2} \\
\hline & & Male & Female & All & Male & Female & All \\
\hline Human & $\operatorname{Max} N$ 's & (195) & (74) & (289) & (195) & $(80)$ & $(300)$ \\
\hline \multirow[t]{6}{*}{ Prodl } & Direct & $-0.34 * *$ & -0.18 & $-0.30^{* *}$ & $0.36^{* *}$ & $0.44^{* *}$ & $0.38 * *$ \\
\hline & Int-Res & $-0.22 * *$ & -0.04 & $-0.17 * *$ & $0.24 * *$ & 0.09 & $0.21 * *$ \\
\hline & Self-Comp & -0.11 & -0.04 & $-0.10^{*}$ & $0.17^{* *}$ & $0.24^{*}$ & $0.18^{* *}$ \\
\hline & Self-Eff & $-0.32 * *$ & -0.17 & $-0.28 * *$ & $0.35^{* * *}$ & $0.42 * *$ & $0.36^{* *}$ \\
\hline & Prsnity & $-0.22^{* *}$ & -0.11 & $-0.19 * *$ & $0.24 * *$ & $0.33^{* * *}$ & $0.26^{* *}$ \\
\hline & All & -0.08 & -0.15 & 0.02 & $0.13^{*}$ & 0.08 & $0.13^{*}$ \\
\hline \multirow[t]{6}{*}{ Prod2 } & Direct & $-0.23 * *$ & 0.05 & $-0.13^{*}$ & $0.18 * *$ & 0.09 & $0.15^{*}$ \\
\hline & Int-Res & $-0.16^{*}$ & 0.15 & -0.05 & $0.12^{*}$ & -0.13 & 0.06 \\
\hline & Self-Comp & -0.05 & 0.17 & 0.02 & 0.03 & -0.07 & 0.00 \\
\hline & Self-Eff & $-0.17 * *$ & 0.06 & -0.10 & $0.13^{*}$ & 0.11 & $0.12 *$ \\
\hline & Prsnlty & $-0.14^{*}$ & 0.11 & -0.05 & $0.10^{*}$ & 0.02 & 0.07 \\
\hline & All & -0.07 & 0.12 & $0.20^{*}$ & 0.04 & -0.15 & -0.01 \\
\hline \multirow[t]{6}{*}{ Prod3 } & Direct & $-0.26 * *$ & $-0.20^{*}$ & $-0.22 * *$ & $0.18^{* *}$ & 0.14 & $0.16^{* *}$ \\
\hline & Int-Res & $-0.21 * *$ & $-0.15^{*}$ & $-0.18 * *$ & 0.10 & -0.03 & 0.06 \\
\hline & Self-Comp & $-0.15^{*}$ & $-0.12^{*}$ & $-0.14^{*}$ & 0.06 & -0.03 & 0.03 \\
\hline & Self-Eff & $-0.25^{* *}$ & -0.18 & $-0.22 * *$ & $0.16^{*}$ & 0.12 & $0.14 *$ \\
\hline & Prsnity & $-0.20^{* *}$ & -0.17 & $-0.18^{* *}$ & 0.09 & 0.06 & 0.08 \\
\hline & All & $-0.13^{*}$ & $-0.33^{*}$ & -0.11 & 0.02 & -0.04 & 0.01 \\
\hline \multirow{7}{*}{$\begin{array}{l}\text { Soc } \\
\text { Sci } \\
\text { Prod } 1\end{array}$} & $\operatorname{Max} N^{\prime} s$ & $(251)$ & (32) & (274) & $(251)$ & (32) & $(318)$ \\
\hline & Direct & $-0.28 * *$ & $-0.44 * *$ & -0.29 & $0.25^{* * *}$ & $0.41^{* *}$ & $0.26^{* *}$ \\
\hline & Int-Res & -0.07 & $-0.38^{*}$ & $-0.38^{*}$ & -0.03 & 0.26 & -0.01 \\
\hline & Self-Comp & -0.04 & -0.23 & -0.05 & -0.01 & 0.19 & $0.15^{*}$ \\
\hline & Self-Eff & $-0.26^{* *}$ & $-0.38^{*}$ & $-0.27^{* *}$ & $0.22 * *$ & $0.38^{*}$ & $0.23 * *$ \\
\hline & Prsnlty & $-0.16^{* * *}$ & -0.25 & $-0.17 * *$ & 0.10 & $0.29 *$ & $0.11^{*}$ \\
\hline & All & 0.03 & -0.24 & 0.00 & $-0.11 *$ & 0.17 & -0.08 \\
\hline \multirow[t]{6}{*}{ Prod2 } & Direct & $-0.17^{* *}$ & $-0.38 * *$ & -0.19 & $0.20^{* *}$ & $0.29 *$ & $0.21^{* *}$ \\
\hline & Int-Res & -0.03 & $-0.30^{*}$ & -0.07 & 0.00 & $0.33^{*}$ & 0.04 \\
\hline & Self-Comp & -0.01 & -0.21 & -0.03 & 0.01 & 0.20 & 0.04 \\
\hline & Self-Eff & $-0.15^{* *}$ & $-0.29 *$ & $-0.16^{* *}$ & $0.14 *$ & $0.30^{*}$ & $0.16 * *$ \\
\hline & Prsnlty & -0.07 & -0.18 & -0.08 & 0.05 & 0.21 & 0.07 \\
\hline & All & 0.03 & -0.17 & 0.00 & -0.05 & 0.27 & -0.01 \\
\hline \multirow[t]{6}{*}{ Prod3 } & Direct & $-0.19 * *$ & $-0.41 * *$ & -0.21 & $0.23^{* *}$ & 0.11 & $0.22^{* *}$ \\
\hline & Int-Res & -0.06 & $-0.33^{*}$ & $-0.10^{*}$ & 0.04 & 0.12 & 0.06 \\
\hline & Self-Comp & -0.05 & -0.23 & -0.08 & 0.08 & -0.04 & 0.08 \\
\hline & Self-Eff & $-0.18 * *$ & $-0.36^{*}$ & $-0.20^{* *}$ & $0.19^{* *}$ & 0.15 & $0.19 * *$ \\
\hline & Prsnlty & -0.10 & -0.20 & $-0.12^{*}$ & $0.10^{*}$ & -0.02 & $0.10^{*}$ \\
\hline & All & -0.01 & -0.20 & -0.04 & 0.01 & 0.01 & 0.02 \\
\hline
\end{tabular}

$*_{p}<.01 ; * p<.05$ 
Turning to the effects of the intervening/moderating variables, with but one exception (out of a possible 72), the moderator reduced the magnitude of the direct effect. For the most part, self-competence and person characteristics (ambitious, competitive, . . .) were the strongest moderators; self-efficacy (influencing publication and securing extra travel dollars) was the weakest. Interest in research (Int-Res) was a strong moderator for humanists.

When all four moderators were included in the analysis, the coefficient reduction generally was the greatest. In 16 of the 18 cases for research universities (Table 2) the correlation between stress and productivity no longer remained significant when all moderators were present. (The numbers are 15 of 18 for Doc-II and Comp-I [Table 4] and 8 of 18 for Doc-I and LA-I [Table 3]). However, interactions exist between the moderators. (See, for example, female natural scientists' collegial conversation output (Prod3) measure for the Stress-1.) Self-competence reduced the direct effect $(-.38)$ to -0.01 (essentially zero), but it rose to -0.07 when all four moderators were included.)

In addition, sex differences exist. Note that for the first productivity measure (publications-Prodl) a large negative correlation for women $(-.51)$ decreases to -.18 (no longer significant) by the interest in research moderator (acting alone) and to -.07 by the self-competence moderator acting alone. Self-efficacy and personality variables fail to significantly reduce the stress effects. If women natural scientists have a high interest in research and feel self-competent in conducting research, the stress of not giving as much time as they desire disappears. ${ }^{6}$ When all moderators are entered, the correlation even turns positive (but $p>.05$ ). ${ }^{7}$ See Table 5 where we display these findings. ${ }^{8}$

Last, as Table 6 reveals, the environmental moderators had essentially no effect on reducing the direct effects of stress. ${ }^{9}$ External grants were the strongest mitigators, but they did not remove the statistical significance. External grants correlate with publication output (Bentley and Blackburn, 1991) but do

TABLE 5. Pearson Product-Moment and Partial Correlations of Job Strains with Research Product Outputs, with and without Personal Moderator Variables

\begin{tabular}{|c|c|c|c|c|c|c|c|}
\hline \multirow[b]{2}{*}{$\begin{array}{l}\text { Research } \\
\text { Products }\end{array}$} & \multirow[b]{2}{*}{$\begin{array}{l}\text { Selected } \\
\text { Moderators }\end{array}$} & \multicolumn{2}{|c|}{$\begin{array}{c}\text { Men } \\
N=266\end{array}$} & \multicolumn{2}{|c|}{$\begin{array}{c}\text { Women } \\
N=37\end{array}$} & \multicolumn{2}{|c|}{$\begin{array}{c}\text { All } \\
N=303\end{array}$} \\
\hline & & $\begin{array}{l}r \text { without } \\
\text { Moderator }\end{array}$ & $\begin{array}{l}r \text { with } \\
\text { Moderator }\end{array}$ & $\begin{array}{l}r \text { without } \\
\text { Moderator }\end{array}$ & $\begin{array}{c}r \text { with } \\
\text { Moderator }\end{array}$ & $\begin{array}{l}r \text { without } \\
\text { Moderator }\end{array}$ & $\begin{array}{c}r \text { with } \\
\text { Moderator }\end{array}$ \\
\hline & $\begin{array}{l}\text { Interest in } \\
\text { Research }\end{array}$ & $-.48^{* *}$ & $-.37^{* *}$ & $-.51 * *$ & -.18 & $-.48 * *$ & $-.35 * *$ \\
\hline Articles, & $\begin{array}{l}\text { Self- } \\
\text { competence }\end{array}$ & $--.48 * *$ & $-.23^{* *}$ & $-.51 * *$ & -.07 & $-.48^{* *}$ & $-.21 * *$ \\
\hline chapters, & Self-efficacy & $-.48 * *$ & $-.46 * *$ & $-.51 * *$ & $-.44 *$ & $-.48 * *$ & $-.44 * *$ \\
\hline books, & Personality & $-.48 * *$ & $-.37 * *$ & $-.51 *$ & $-.36^{*}$ & $-.48^{* *}$ & $-.36^{* *}$ \\
\hline etc. & All moderators & $-.48 * *$ & $-.21^{* *}$ & $-.51 * *$ & +.13 & $-.48 * *$ & $-.19 * *$ \\
\hline
\end{tabular}

${ }^{*} p<.05 ; * * p<.01$ 
TABLE 6. Environmental Variables (Research I \& II): Direct and Partial Effects

\begin{tabular}{|c|c|c|c|c|c|c|c|}
\hline \multirow{2}{*}{ Nat } & \multirow[b]{3}{*}{$\operatorname{Max} N^{\prime} \mathrm{s}$} & \multicolumn{3}{|c|}{ Stress 1} & \multicolumn{3}{|c|}{ Stress 2} \\
\hline & & Male & Female & All & Male & Female & All \\
\hline Sci & & (189) & (21) & (213) & $(189)$ & $(21)$ & $(213)$ \\
\hline \multirow[t]{6}{*}{ Prod 1} & Direct & $-0.48 * *$ & $-0.51 * *$ & $-0.48 * *$ & $0.34 * *$ & $0.41^{*}$ & $0.34 * *$ \\
\hline & Support & $-0.50 * *$ & $-0.49 * *$ & $-0.49 * *$ & $0.38 * *$ & $0.51^{* *}$ & $0.38 * *$ \\
\hline & Assist & $-0.50^{* *}$ & $-0.50^{* *}$ & $-0.50^{* *}$ & $0.36^{* * *}$ & $0.55^{* *}$ & $0.37 * *$ \\
\hline & Ingrant & $-0.45 * *$ & $-0.48 * *$ & $-0.49 * *$ & $0.33^{* *}$ & $0.51^{* *}$ & $0.33 * *$ \\
\hline & Extgrant & $-0.39 * *$ & $-0.48 * *$ & $-0.39 * *$ & $0.25^{* *}$ & $0.50^{* *}$ & $0.27 * *$ \\
\hline & All & $-0.42 * *$ & $-0.50^{* *}$ & $-0.42^{* *}$ & $0.27^{* *}$ & $0.57 * *$ & $0.29 * *$ \\
\hline \multirow[t]{6}{*}{ Prod2 } & Direct & $-0.33^{* *}$ & -0.26 & $-0.33^{* *}$ & $0.34 * *$ & 0.06 & $0.31 * *$ \\
\hline & Support & $-0.37 * *$ & -0.21 & $-0.35^{* *}$ & $0.36^{* *}$ & 0.19 & $0.34 * *$ \\
\hline & Assist & $-0.35^{* *}$ & -0.21 & $-0.34^{* *}$ & $0.34 * *$ & 0.16 & $0.32 * *$ \\
\hline & Ingrant & $-0.31 * *$ & -0.20 & $-0.30 * *$ & $0.31^{* *}$ & 0.14 & $0.28 * *$ \\
\hline & Extgrant & $-0.27 * *$ & -0.21 & $-0.26 * *$ & $0.25^{* *}$ & 0.16 & $0.24 * *$ \\
\hline & All & $-0.28 * *$ & -0.20 & $-0.27 * *$ & $0.28 * *$ & 0.16 & $0.27 * *$ \\
\hline \multirow[t]{6}{*}{ Prod3 } & Direct & $-0.43 * *$ & $-0.38 *$ & $-0.42 * *$ & $0.42 * *$ & 0.22 & $0.40^{* *}$ \\
\hline & Support & $-0.44 * *$ & -0.34 & $-0.43 * *$ & $0.43 * *$ & 0.28 & $0.42 * *$ \\
\hline & Assist & $-0.44^{* *}$ & $-0.34^{*}$ & $-0.43^{* *}$ & $0.43^{* *}$ & 0.28 & $0.41 * *$ \\
\hline & İngrant & $-0.39 * *$ & $-0.35^{*}$ & $-0.39 * *$ & $0.40^{* *}$ & 0.28 & $0.38 * *$ \\
\hline & Extgrant & $-0.33^{* *}$ & $-0.37^{*}$ & $-0.34 * *$ & $0.33 * *$ & 0.29 & $0.33^{* *}$ \\
\hline & All & $-0.33^{* *}$ & $-0.39 *$ & $-0.34^{* *}$ & $0.34^{* *}$ & $0.37^{*}$ & $0.34^{* *}$ \\
\hline Human & $\operatorname{Max} N^{\prime} \mathrm{s}$ & (114) & (36) & (153) & (114) & $(36)$ & $(153)$ \\
\hline \multirow[t]{6}{*}{ Prodl } & Direct & $-0.25^{* *}$ & $-0.31^{*}$ & $-0.25 * *$ & $0.32 * *$ & $0.32 * *$ & $0.29 * *$ \\
\hline & Support & $-0.23 * *$ & $-0.38 * *$ & $-0.25 * *$ & $0.26^{* *}$ & $0.30 *$ & $0.26^{* *}$ \\
\hline & Assist & $-0.27 * *$ & $-0.40 * *$ & $-0.27 * *$ & $0.27^{* *}$ & $0.32 *$ & $0.26^{* *}$ \\
\hline & Ingrant & $-0.24 * *$ & $-0.37^{* *}$ & $-0.25^{* *}$ & $0.25^{* *}$ & $0.30^{*}$ & $0.25^{* *}$ \\
\hline & Extgrant & $-0.22 * *$ & $-0.40^{* *}$ & $-0.25 * *$ & $0.22 * *$ & $0.31 *$ & $0.23 * *$ \\
\hline & All & $-0.21 * *$ & $-0.43^{* *}$ & $-0.23 * *$ & $0.19^{*}$ & $0.34^{*}$ & $0.21 * *$ \\
\hline \multirow[t]{6}{*}{ Prod2 } & Direct & $-0.29 * *$ & 0.00 & $-0.18 * *$ & $0.26^{* *}$ & 0.02 & $0.18^{* *}$ \\
\hline & Support & $-0.32 * *$ & -0.03 & $-0.2 l^{* *}$ & $0.29 * *$ & 0.01 & $0.17^{*}$ \\
\hline & Assist & $-0.30^{* *}$ & 0.06 & $-0.18^{* *}$ & $0.25^{* *}$ & -0.04 & $0.15 *$ \\
\hline & Ingrant & $-0.25^{* *}$ & 0.03 & $-0.16^{*}$ & $0.20^{*}$ & 0.03 & 0.12 \\
\hline & Extgrant & $-0.25^{* *}$ & 0.02 & $-0.16^{*}$ & $0.19 *$ & 0.02 & 0.11 \\
\hline & All & $-0.27 * *$ & 0.01 & $-0.17 *$ & $0.20^{*}$ & 0.00 & 0.12 \\
\hline \multirow[t]{6}{*}{ Prod3 } & Direct & $-0.25^{* *}$ & $-0.40^{* *}$ & $-0.22 * *$ & $0.32 * *$ & $0.33 * *$ & $0.28 * *$ \\
\hline & Support & $-0.29 * *$ & $-0.51 * *$ & $-0.26^{* *}$ & $0.28 * *$ & $0.42 * *$ & $0.25 * *$ \\
\hline & Assist & $-0.25^{* *}$ & $-0.42 * *$ & $-0.21^{* *}$ & $0.22^{* *}$ & $0.37 * *$ & $0.21 * *$ \\
\hline & Ingrant & $-0.21 * *$ & $-0.47 * *$ & $-0.20^{* *}$ & $0.18^{*}$ & $0.40^{* *}$ & $0.18 * *$ \\
\hline & Extgrant & $-0.20^{*}$ & $-0.47 * *$ & $-0.19 * *$ & $0.16^{*}$ & $0.40^{* *}$ & $0.17^{*}$ \\
\hline & All & $-0.24 * *$ & $-0.53^{* *}$ & $-0.23^{* *}$ & $0.21^{* *}$ & $0.43^{* *}$ & $0.20^{* *}$ \\
\hline
\end{tabular}

Soc

Sci

$\operatorname{Max} N$ 's $\quad(168)$

(40)

(208)

(168)

(40)

(208) 


\begin{tabular}{rlllllll} 
Prodl & Direct & $-0.32^{* *}$ & $-0.53^{* *}$ & $-0.35^{* *}$ & $0.30^{* *}$ & $0.52^{* *}$ & $0.32^{* *}$ \\
& Support & $-0.30^{* *}$ & $-0.46^{* *}$ & $-0.32^{* *}$ & $0.29^{* *}$ & $0.47^{* *}$ & $0.31^{* *}$ \\
& Assist & $-0.28^{* *}$ & $-0.43^{* *}$ & $-0.29^{* *}$ & $0.28^{* *}$ & $0.53^{* *}$ & $0.30^{* *}$ \\
& Ingrant & $-0.28^{* *}$ & $-0.47^{* *}$ & $-0.30^{* *}$ & $0.28^{* *}$ & $0.50^{* *}$ & $0.30^{* *}$ \\
& Extgrant & $-0.24^{* *}$ & $-0.46^{* *}$ & $-0.27^{* *}$ & $0.26^{* *}$ & $0.48^{* *}$ & $0.28^{* *}$ \\
& All & $-0.22^{* *}$ & $-0.39^{* *}$ & $-0.24^{* *}$ & $0.24^{* *}$ & $0.51^{* *}$ & $0.26^{* *}$ \\
Prod2 & Direct & $-0.21^{* *}$ & $-0.28^{*}$ & $-0.22^{* *}$ & $0.14^{*}$ & 0.16 & $0.16^{* *}$ \\
& Support & -0.19 & $-0.33^{*}$ & $-0.20^{* *}$ & $0.15^{*}$ & 0.23 & $0.16^{* *}$ \\
& Assist & -0.17 & -0.15 & $-0.17^{* *}$ & $0.13^{*}$ & 0.16 & $0.13^{*}$ \\
& Ingrant & $-0.16^{*}$ & -0.22 & $-0.17^{* *}$ & $0.12^{*}$ & 0.15 & $0.12^{*}$ \\
& Extgrant & $-0.13^{*}$ & -0.21 & $-0.14^{*}$ & 0.10 & 0.12 & 0.10 \\
& All & -0.12 & -0.24 & $-0.13^{*}$ & 0.10 & 0.25 & 0.11 \\
& Direct & $-0.23^{* *}$ & $-0.34^{* *}$ & $-0.24^{* *}$ & $0.22^{* *}$ & -0.02 & $0.21^{* *}$ \\
& Support & $-0.23^{* *}$ & -0.14 & $-0.22^{* *}$ & $0.28^{* *}$ & -0.07 & $-0.24^{* *}$ \\
& Assist & $-0.18^{* *}$ & -0.16 & $-0.18^{* *}$ & $0.23^{* *}$ & 0.03 & $0.20^{* *}$ \\
& Ingrant & $-0.18^{* *}$ & -0.20 & $-0.17^{* *}$ & $0.22^{* *}$ & 0.03 & $0.19^{* *}$ \\
& Extgrant & $-0.16^{*}$ & -0.17 & $-0.16^{* *}$ & $0.22^{* *}$ & -0.02 & $0.18^{* *}$ \\
& All & $-0.16^{*}$ & -0.03 & $-0.15^{*}$ & $0.24^{* *}$ & -0.10 & $0.19^{* *}$ \\
\hline
\end{tabular}

$* * p<.01 ; * p<.05$

not alter stress. Institutional grants are held by relatively few faculty and alter conditions slightly for the majority. Stress persists even when one has assistance and supportive colleagues-unless one has the right personal attributes.

\section{DISCUSSION/IMPLICATIONS}

Aggregating institutional types into a larger category and combining already subspecialized academic disciplines into fields of knowledge introduces unknown limitations in the findings. There may be differences between Doc-I and LA-I institutions and between mathematicians and biologists that aggregated data suppress. The individual nature of research in the humanities and the team approach in the sciences suggest there may be other differences we failed to uncover. Also, environmental variables have less variance and hence will have less statistical power. HLM (Hierarchical Linear Modeling) can test this possibility, but the procedure requires larger $N$ 's than we have. We noted other limitations in the text.

We can speculate on the reasons for the observed differences when we make comparisons across the disciplines and place of work (institutional types) and between the sexes. The higher coefficients in the natural sciences are in accord with the reputation these fields have. Big science is where big money exists, where the prizes for successful discoveries invite cut-throat competition, where fraud makes the headlines. High stakes exist for the players in this game. Stress can also be predicted to be at a higher level. 
That the stress levels tend to be higher in the R-I, R-II category has an analogous explanation. These are the institutions that place the strongest value on research, the ones that compete with one another for external funding, for reputational standing in the national rankings of departments, and for collecting as many national and international faculty stars as they can afford. Those who choose to work in the research universities can be expected to have higher selfimposed levels of stress than faculty in other institutional types.

Women, underrepresented everywhere, are most markedly absent in the sciences and in R-I and -II universities. Their percentages of the work force are lowest in these disciplines and institutions. To be successful, as these women are, their interest in publishing research and their competence to conduct successful research have to be extraordinarily high in order to survive in these male-dominated fields. Not surprising, then, is that the possession of the personal attributes of high interest in research and strong self-competence are effective mitigators of stress for these female faculty.

As for the theoretical outcomes, the results provide overall support for the Harrison person-environment fit theory and the Lawrence et al. cognitive motivation model on which this inquiry was based. In particular, the Lawrence model suggested the variables that most significantly reduced strains. Faculty perceptions of self are important and strong predictors of research output.

On the practical side, if an institution wants to increase research output but not raise faculty stress to a dysfunctional level, it needs to examine the possibilities and costs of interventions. Environmental variables are more easily changed than are personal attributes, but, as seen, the ones used here are not effective. A supportive climate has not removed the strains faculty express as a consequence of not allocating their research effort in either their personal preferred way or in their perceptions of what the institution desires.

Within the self knowledge variables, changing personality characteristics and skills is no easy matter. Attempting to do so can even be hazardous. One cannot easily convert a type B person into a highly competitive individual with an increased interest in research, at least not at the outset. Still, administrators can provide ways of enhancing faculty research skills, thereby increasing competence, a high mitigator of stress. Improved research talent may, in turn, lead to greater research success, which, in turn, could lead to higher self-competence and interest in research. Both mitigate the dysfunctional consequences of stress. At the same time, attempting to induce these involves genuine risks. The faculty member may agree to participate in a program with these goals but may fail to gain the competence needed to succeed. Personal harm can result.

As the Harrison model shows (Figure 1 above), coping is another way to deal with stress. The institution could invest in experts to aid faculty in coping strategies. Also, informal reports suggest that pairing - and supporting-less productive faculty with high producers increases the former's output. Chairs have been shown to be successful interventionists (Creswell et al., 1987). 
Returning to the theoretical side, one might also test for the effectiveness of other environmental variables. Stress, qua stress, is not going to abandon faculty lives.

Acknowledgments. We thank Professor John Creswell, University of Nebraska-Lincoln, for his thoughtful and constructive critique of an earlier version of this manuscript.

\section{NOTES}

1. Administration's heightened demands for a greater research output are not accompanied by reductions in other faculty role expectations. While we know of no empirical data, we universally hear that just the opposite is the case. Faculty are simultaneously urged to give more time to students and to improve their teaching.

2. The subsequent discussion uses Boberg (1982:7-11).

3. Unfortunately, their findings are not linked to a productivity outcome variable such as articles produced.

4. Some might believe the products should receive differential weights because of their varying length of quality. Weighting scales have been advanced (see, e.g., Stallings and Singhal, 1970). However, what has been learned is the weighted measure of research does not significantly change the order of the producers. That is, the correlations generated are essentially identical with weighted or unweighted scores. This is not surprising. As an indicator of the research construct--either a single item (e.g., how many publications in the last two years, the standard in the survey field of faculty research output), and hence of no reliability or a factor or scale of multiple items with high reliability - both fail to capture the full meaning of "research."

5. The three measures are correlated with one another.

6. Trautvetter and Blackburn (1990) show with comparative regressions for men and women that the most salient predictor of women's article output is their self-assessed scholarly competence.

7. That there are somewhat fewer significant correlations for women than men is partly an artifact of the N's. Appreciably fewer number of women exist in every instance (by category and discipline) and hence the $r$ 's need to be larger in order to attain the same statistical significance level. The small $N$ 's for women was the reason for grouping institutional types.

8. All 18 cells in Table 2 could be presented in this more readable form. However, to do so for all three institutional categories and two moderating type variables requires 108 tables. The interested reader should take any of the cells in Tables 2, 3, 4, and 6 and put them in this format for easier reading and analysis. We apologize for the massive amount of data contained in these four tables and the difficulty of dealing with them.

9. Similar none results exist for the other two institutional categories so there is no need to include these tables.

\section{REFERENCES}

Argyris, C. (1964). Integrating the Individual and the Organization. New York: John Wiley.

Bentley, R. J., and Blackburn, R. T. (1991). Changes in academic research performance over time: A study of institutional accumulative advantage. Research in Higher Education 31(4):327-323.

Bieber, J. P., and Blackburn, R. T. (1989, November). Faculty research productivity 1972-1988: Development and application of constant units of measure. Paper pre- 
sented at the annual meeting of the Association for the Study of Higher Education, Atlanta. (Now substantially revised.)

Blackburn, R. T., Bieber, J. P., Lawrence, J. H., and Truatvetter, L. C. (1991). Faculty at work: Focus on research, scholarship, and service. Research in Higher Education 32(4):385-415.

Blackburn, R. T., Horowitz, S. M., Edington, D. W., and Klos, D. (1987). University faculty and administrator responses to job strains. Research in Higher Education 25(1):31-41.

Blackburn, R. T., and Mackie, C. J. (1992). Test-retest coefficients and the national faculty surveys. Review of Higher Education 16(1):19-39.

Boberg, A. D. (1982). Faculty under stress: Person-environment fit theory. Unpublished doctoral dissertation, University of Michigan.

Campbell, D. B. ( 1973). A program to reduce coronary heart disease risk by altering job stresses. Unpublished doctoral dissertation, University of Michigan.

Carnegie Foundation for the Advancement of Teaching (1987). A Classification of Institutions of Higher Education. Princeton, NJ: Carnegie Foundation.

Clark, M. J., and Blackburn, R. T. (1973). Faculty performance under stress. In A. L. Sockloff (ed), Faculty Effectiveness as Evaluated by Students, (pp. 233-252). Philadelphia: Temple University.

Creswell, J. W., Seagren, A., Wheeler, D., Vavrus, L., Grady, M., Egly, N., and Wilhite, M. (1987, November). The faculty development role of department chairs: A naturalistic analysis. Paper presented at the annual meeting of the Association for the Study of Higher Education: Baltimore, MD.

Dey, E. L. (1990, November). Dimensions of faculty stress: Evidence from a recent national survey. Paper presented at the annual meeting of the Association for the Study of Higher Education: Portland, OR.

French, J. R. P., Caplan, R. D., and Harrison, R. V. (1982). The Mechanisms of Job Stress and Strain. New York: Wiley.

Gmelch, W. H., Lovrich, N. P., and Wilke, P. K. (1984), Sources of stress in academe: A national perspective. Research in Higher Education 20(4):477-490.

Gmelch, W. H., Wilke, P. K., and Lovrich, N. P. (1986). Dimensions of stress among university faculty: Factor-analytic results from a national study. Research in Higher Education 24(3):266-286.

Harrision, R. V. (1976). Job demands and worker health: Person-environment misfit. Unpublished doctoral dissertation, University of Michigan.

House, J. S. (1972). The relationship of intrinsic and extrinsic work motivations to occupational stress and coronary heart disease risk. Unpublished doctoral dissertation, University of Michigan.

Keinan, G., and Perlberg, A. (1987). Stress in academe: A cross-cultural comparison between Israeli and American academicians. Journal of Cross-Cultural Psychology 18(2):193-207.

Lawler, E. E. (1973). Motivation in Work Organizations. Belmont, CA: Wadsworth.

Lawrence, J. H., Frank, K., Blackburn, R. T., Bieber, J. P., Bentley, R. J., and Trautvetter, L. C. (1989, March). Faculty scholarly output: Development of a theoretical model. Paper presented at the annual meeting of the American Educational Research Association, San Francisco.

Lewin, K. (1951). Field Theory in Social Science. New York: Harper \& Row.

Locke, E. A. (1969). What is job satisfaction? Organizational Behavior and Human Performance 4:309-366.

Pelz, D. C., and Andrews, F. M. (1976). Scientists in Organizations. New York: Wiley. 
Rotherman, M. J., \& Weiner, N. (1983). Androgyny, stress, and satisfaction: Dualcareer and traditional relationships. Journal of Research 9(2):151-158.

Stallings, W. M., and Singhal, S. (1970). Some observations on the relationships between research productivity and student evaluations of courses and teaching. American Sociologist 5:141-143.

Trautvetter, L. C., and Blackburn, R. T. (1990, April). Gender differences in predicting faculty publication output in the natural sciences. Paper presented at the annual meeting of the American Educational Research Association, Boston.

Zuckerman, H. (1991). The careers of men and women scientists: A review of current research. In Harriet Zuckerman, Jonathan Cole, and John Bruer (eds.), The Outer Circle: Women's Position in the Scientific Community. New York: W. W. Norton.

Zuckerman, H., Cole, J. R., and Bruer, J. T. (1991). The Outer Circle: Women in the Scientific Community. New York: W. W. Norton

Received June 18, 1992. 